\title{
Systematic review and meta-analysis of the clinical survival significance of Sialyl-Tn expression in histological tissues from cancer patients
}

\author{
Roberto Lugo, Azalia Ávila-Nava, Rodrigo García-Pérez, Sarahí Herrera-Escalante, Jocelyne De la Cruz- \\ Acosta, Ana Ligia Gutiérrez-Solis \\ Regional High Speciality Hospital of the Yucatan Peninsula, Merida, Yucatan, México \\ Contributions: (I) Conception and design: AL Gutiérrez-Solis; (II) Administrative support: R Lugo; (III) Provision of study materials or patients: R \\ García-Pérez, S Herrera-Escalante, J De la Cruz-Acosta; (IV) Collection and assembly of data: AL Gutiérrez-Solis, R Lugo, A Ávila-Nava; (V) Data \\ analysis and interpretation: AL Gutiérrez-Solis; (VI) Manuscript writing: All authors; (VII) Final approval of manuscript: All authors. \\ Correspondence to: Ana Ligia Gutiérrez-Solis, PhD. Hospital Regional de Alta Especialidad de la Península de Yucatán, Calle 7, No. 433 por 20 y 22, \\ Fraccionamiento Altabrisa, Mérida, Yucatán, México, 97130. Email: ganaligia@gmail.com.
}

\begin{abstract}
Background: The expression of Sialyl-Tn (STn) has been associated with different mechanisms of cancer. Several studies using histological tissue samples from different types of cancer showed differences in the STn expression patterns, but few studies have explored the association between STn expression and decreased overall survival. Therefore, a comprehensive analysis of the clinical significance of STn in cancer patients was performed.
\end{abstract}

Methods: A systematic review and meta-analysis were done of scientific articles published and available from different sources, including MEDLINE/PubMed, Web of Science, Cochrane Library and LILACS. Odds ratio (OR) was represented by the number of deaths during follow-up in positive-STn versus negativeSTn patients. OR from each study was pooled and the results were presented in a forest plot.

Results: Eleven articles were included in the systematic meta-analysis. The estimated OR of 3.26 (95\% CI: 2.12-5.02) was obtained. Furthermore, subgroups studies of gastric cancer showed an OR of 3.74 (95\% CI: 2.47-5.66) and other cancer types reported an OR of 2.92 (95\% CI: 1.16-7.32).

Conclusions: This study showed that survival time was significantly longer in patients with negative-STn tumors than in those with positive results.

Keywords: Sialyl-Tn (STn); cancer; survival; meta-analysis

Submitted Aug 12, 2019. Accepted for publication Oct 31, 2019.

doi: $10.21037 /$ tcr.2019.11.53

View this article at: http://dx.doi.org/10.21037/tcr.2019.11.53

\section{Introduction}

It has been shown that abnormal glycosylation plays an important role during the carcinogenesis and can lead to the overexpression of mucin-associated carbohydrates as Sialyl-Tn (STn) antigen (1). The biosynthesis of STn involves different pathways, first the Tn antigen is produced through the addition of $\mathrm{N}$-acetylgalactosamine in serine or threonine residues of a mucin protein. Once synthesized, Tn antigen suffers modifications that bounds to different structures such as STn antigen (Sa $2,6 \mathrm{GalNAc} \alpha-\mathrm{O}-\mathrm{Ser} /$
Thr) by addition of sialic acid, antigen $\mathrm{T}$ by addition of galactose (Gal $\beta 1,3$ GalNAc $\alpha-O-S e r / T h r)$ and Core 3 by addition of $\mathrm{N}$-acetylglucosamine (2).

Over the last 40 years, research efforts were focused mainly on undercovering the role of STn antigen during cancer development. Through the $80 \mathrm{~s}$ and 90 s, authors aimed to describe the STn expression in different types of cancers and its clinical use (3). The results showed that expression of STn can be associated with different mechanisms of cancer, but mainly involved in the tumor 


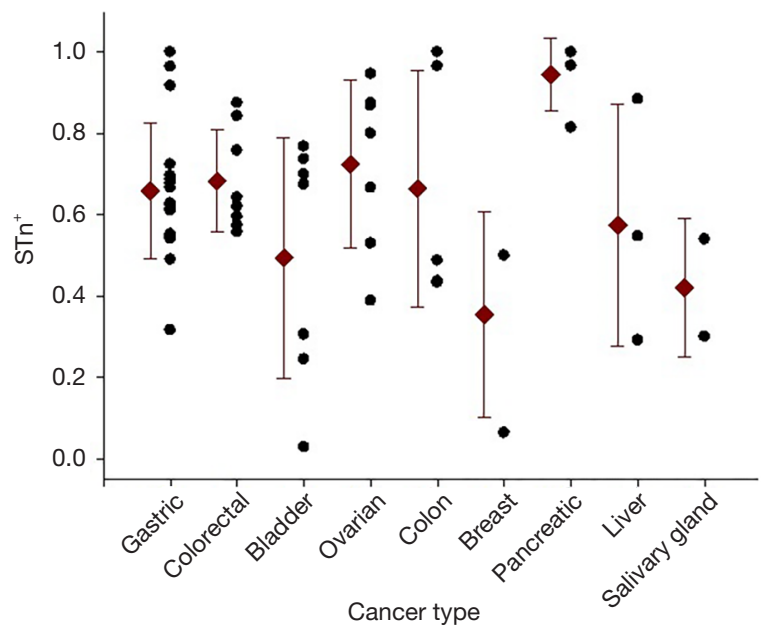

Figure 1 STn frequency in soft tissue carcinomas. Each dot represents a report.

development (4). However, the activation of pathways can depend on the cancer type or subtype (5). These results brought the idea of using immunotherapy, based on antiSTn vaccine, as an attractive approach for the treatment of cancer patients. Therefore, in the early 2000s, some clinical trials (6) were developed in order to explore the potential of the anti-cancer vaccine targeting STn but, despite the great effort involved in the application of the vaccine, the clinical trial failed in phase III (3). Still, taking in consideration the positive and negative results obtained in the past, and the new information that has emerged in the last decade (7), it can be concluded that STn is an attractive target to be revised and contemplated in the design of novel immunotherapies.

Several studies using histological tissue samples from different types of cancer showed differences in the STn expression patterns (Figure 1). Reported data from histological tissues noted consistent high frequencies in colorectal (8), colon (9), gastric (10) and pancreatic cancer (11). Other studies registered some variability in the expression of STn, as for example in breast (12), ovarian (13), bladder (14), liver (15) and cervical cancer (16). In contrast, in normal tissues, STn expression is found to be rare or low compared to the matching cancer cells (17). This heterogeneity can be due to several factors including antibodies used, staining protocol, number of samples evaluated, among others. Many monoclonal antibodies have been developed for STn detection, however B72.3 and TKH2 are the most commonly used anti-STn antibodies according to the literature (18). Overall, it can be concluded that STn expression is higher in the majority of cancers than in healthy tissues. In fact, STn has been postulated as a good tumor marker of carcinogenesis and potentially useful for diagnosis.

In the literature above, only a few studies have explored the association between STn expression and decreased overall survival, but contradictory results can be found among cancer types and subtypes. Therefore, more studies are needed in order to obtain reliable and accurate information regarding the prognostic value of STn in cancer. The very few data available assessing the prognostic value of STn antigen in carcinomas drove us to determine the association between the expression of this mucinassociated carbohydrate antigen and patients survival. For this reason a comprehensive analysis of the clinical significance of STn in cancer patients was performed using a systematic literature review and meta-analysis.

\section{Methods}

\section{Study design and search strategy/data source}

A systematic review was performed independently by tree investigators (ALGS, RL, AAN) using published articles on the clinical significance of the expression of STn in histological tissue samples from cancer patients. The studies were identified through searching in English terms using the online sources of MEDLINE/PubMed, Web of Science, Cochrane Library and LILACS. The grey literature was searched using Google Scholar. The following keywords were taken from the Medical Subject Headings library of PubMed and used in combination: "Sialyl-Tn antigen" AND "Neoplasms" AND "Mortality". The search strategy for Medline was developed first and then adapted for the remaining sources. The search was done for titles and/ or abstracts and was carried out between August 2018 and February of 2019.

\section{Selection of studies}

Three investigators selected the studies for the systematic review and meta-analysis if they reported on the expression of STn using histological tissue samples from carcinoma samples. A total of 108 relevant studies were identified and exported to EndNote X9 (Philadelphia, USA). After removing duplicates, 79 studies were considered as candidates for this review. Then, the following exclusion 

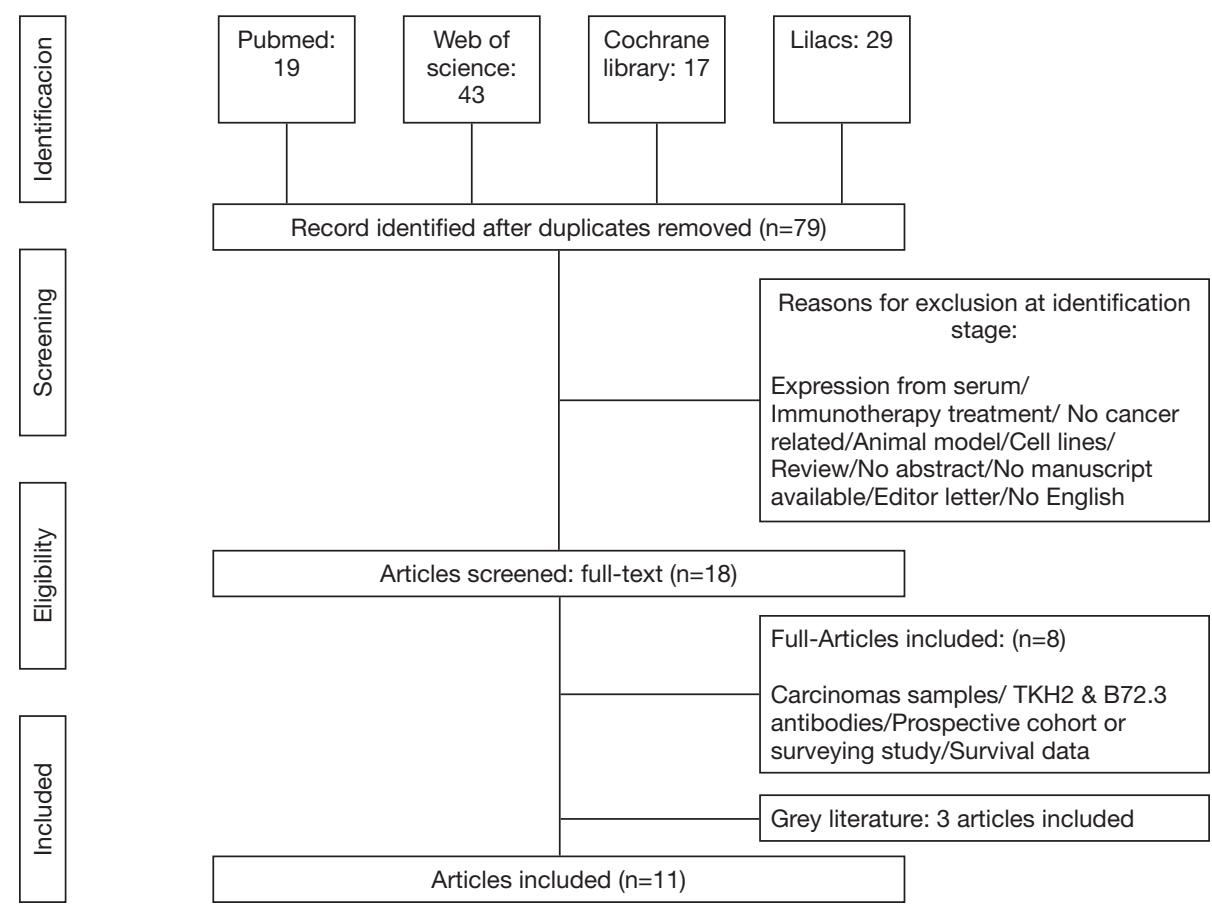

Figure 2 Flow diagram (PRISMA) of selected studies in the systematic review.

criteria were applied: (I) STn expression from serum; (II) studies evaluating immunotherapy; (III) studies from cell lines or animal models; (IV) reviews and letters to the editors; (V) manuscripts without abstract; (VI) no manuscript available. The inclusion criteria for our study was: (I) STn expression from formalin-fixed, paraffinembedded specimens of carcinomas samples; (II) using TKH2 or B72.3 monoclonal antibodies; (III) including survival and mortality data; (IV) prospective cohort or retrospective study design; (V) studies with data of at least five-years follow-up (Figure 2).

After preliminary screening of the titles of the studies, all abstracts were evaluated for eligibility (some studies were assessed in full length), based on the established criteria. There was no restriction in the year of publication, recruitment period or sample size.

\section{Data extraction}

The present study was done based on the criteria of Preferred Reporting Items for Systematic Reviews and Meta-Analysis (PRISMA) (Figure 2). Data from all the selected articles were extracted independently by two investigators (ALGS and RL) into a predefined database, including first author information, year of publication, sample type and size, antibody used, percentage of STn positive and negative (STn+ and STn-) expression, survival data and follow-up of patients (Table 1). GetData Graph Digitizer 2.26 was used to extract survival data from studies that only reported the analysis by Kaplan-Meier graphs; mortality was defined as a death of the patient or recurrence of the disease in period of time of 5 years. In case of discrepancy among investigators, the final consensus was done by another investigator (AAN). The quality of the methodology used in each study was evaluated using The Newcastle-Ottawa Scale (NOS) (19) (Table S1).

\section{Statistical analysis}

Odds ratio (OR) was represented by the number of deaths during follow-up in STn+ versus STn- patients. Five-year follow-up data were extracted; otherwise, the maximum reported follow-up was considered. All statistical analyses were undertaken using the software RStudio version 1.0.153. OR for mortality, comparing STn+ with STn-, was pooled using a random-effects model and afterwards presented in a forest plot with corresponding $95 \%$ confidence interval (CI). Statistical heterogeneity was assessed using the $\mathrm{I}^{2}$ index. Finally, publication bias was assessed by visual inspection of funnel plots and with the Egger bias test (20). 


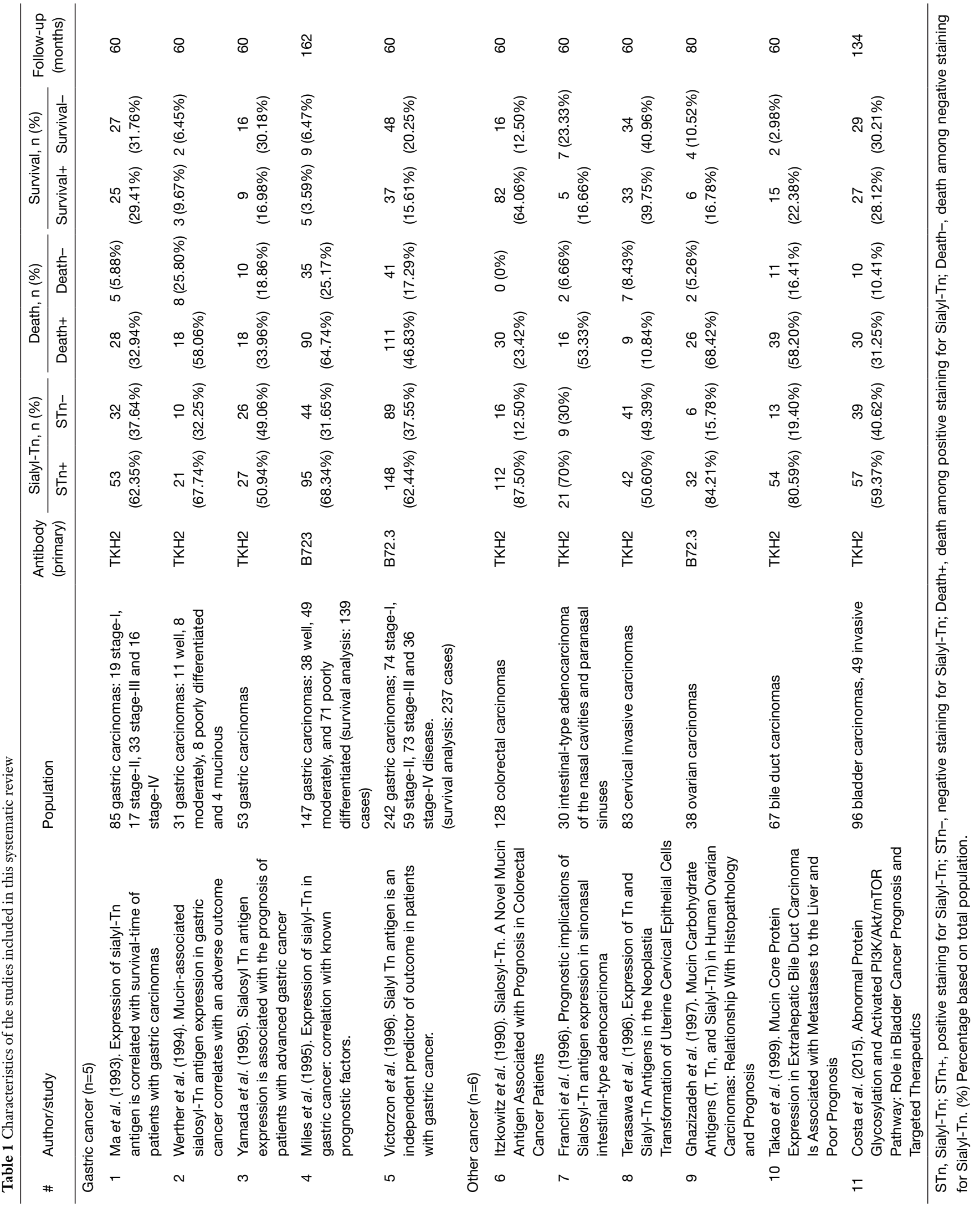




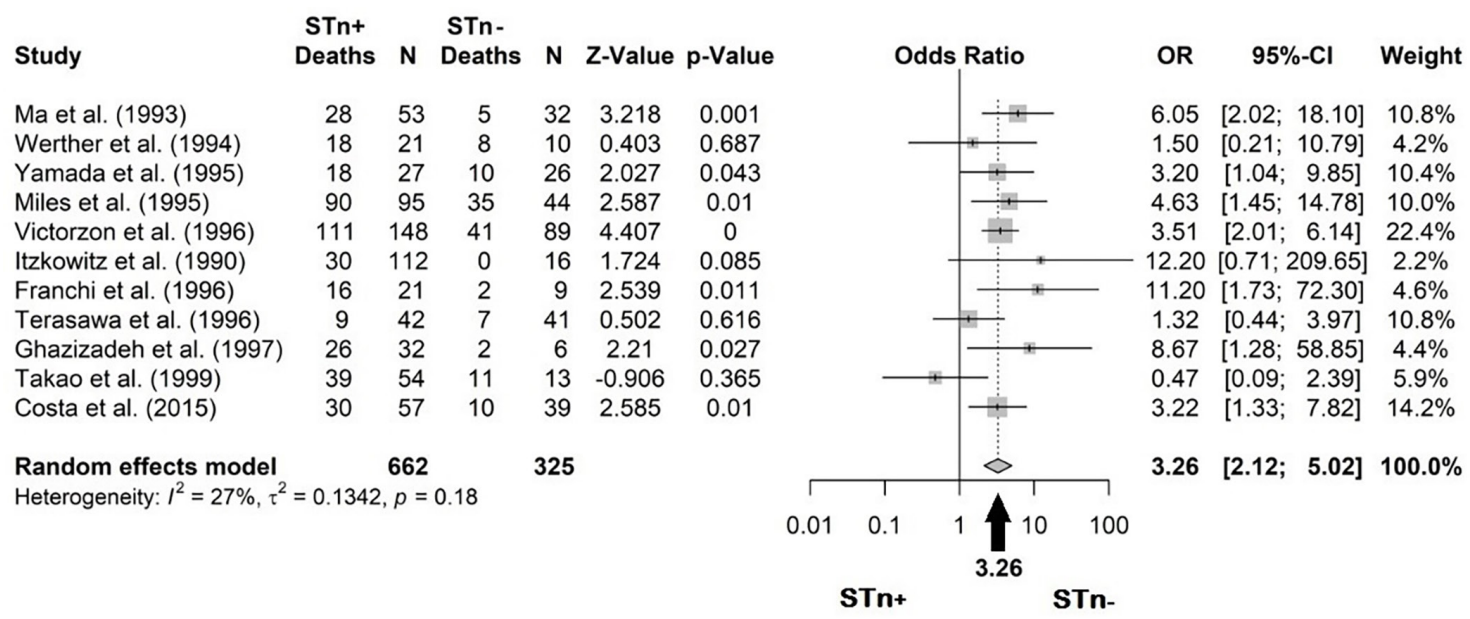

Figure 3 Forest plot of the odds ratio (OR) mortality (STn+ vs. STn-) in cancer patients: gastric (5,21-24); colorectal (9); sinosal (25); cervical (16); ovarian (13); liver (15) and bladder (14).

\section{Results}

\section{Identified studies}

The conducted literature search displayed a total of 108 articles. Once duplicates were eliminated, 79 relevant records were identified and their tittles and abstracts reviewed. After applying the inclusion and exclusion criteria, 11 articles were included for this systematic review and meta-analysis (Figure 2 and Table 1).

\section{Characteristics of studies}

Eleven articles reporting on the association between positive-STn staining in histological tissue samples and cancer patient's overall survival were included. The year of publication of the studies ranged from 1990 to 2015, therefore, the recruitment period of the participants was different across the selected studies. Five of eleven articles were carried out in Japan $(13,15,16,21,22)$ and two in United Stated of America (8,23). Additionally, single studies were performed in Finland (5), Portugal (14), United Kingdom (24) and Italy (25).

All articles assessed the expression of STn by immunohistochemical staining using monoclonal antibodies (TKH2 or B72.3). Carcinoma tumors analyzed ranged from stage I to IV, and included samples from primary tumors and metastatic lesions. Depending of the cancer type, the results were organized in the following categories: five studies of gastric cancer (5,21-24); one study of colorectal cancer (8); one of bladder cancer (14); one of ovarian cancer (13); one of sinonasal cancer (25); one of cervical cancer (16), and finally one of liver cancer (15). A total of 662 patients samples were positive and 325 patients were negative for STn.

The mortality in cancer patients was determined by the percentage of deaths in the STn+ population and in the STn- one. Among gastric cancer patients, mortality or recurrence was found in $77.03 \%(265 / 344)$ patients with STn+ and $46.77 \%(94 / 201)$ that were STn-. In colorectal cancer, $26.79 \%$ (30/112) with STn-positive staining and no deaths were found in patients with STn-negative (0/16). Mortality of $52.63 \%(30 / 57)$ among STn+ expression group was reported in bladder cancer patients and $25.64 \%$ (10/39) with STn-; in ovarian cancer 26 deaths were found among patients with STn-positive and 2 more with STnnegative $(81.25 \%$ and $33.33 \%$, respectively). In sinonasal cancer, $76.19 \%$ (16/21) were positive for STn and $22.22 \%$ (2/9) were negative for STn; and for cervical cancer, it was reported $9 / 42$ deaths with positive and 7/41 with negative staining for STn $(21.42 \%$ vs. $17.07 \%)$. In liver cancer, both positive (39/54) and negative (11/13) STn expressing patients, showed high mortality rates $(72.22 \%$ vs. $84.61 \%)$ (Table 1 and Figure 3).

\section{OR of survival}

An OR of 3.26 was obtained, when all studies among different types of cancers were pooled, meaning that those patients positive for STn have three times higher probability of death comparing with those that are negative 


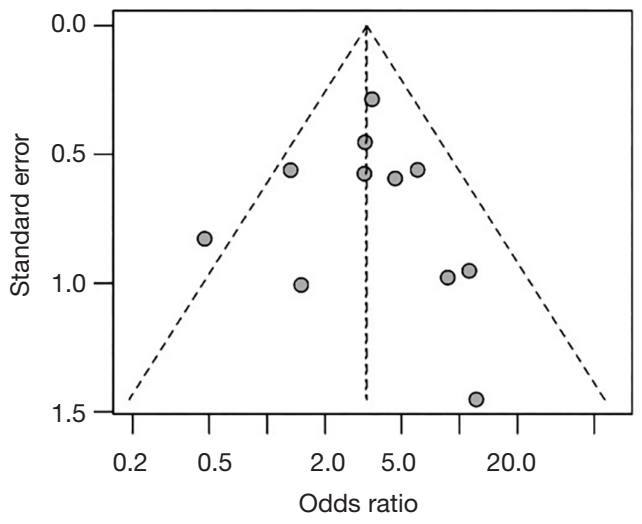

Figure 4 Funnel plot of the odds ratio (OR) mortality for this systematic review and meta-analysis.
(OR: 3.26; 95\% CI: 2.12-5.02) (Figure 3). No publication bias was found (Egger's test $=0.12 ; \mathrm{P}=0.89$ ), as also shown by the funnel plot (Figure 4). Following the general analysis, cancer subgroups were analyzed. When studies reporting gastric cancer were grouped, the results exposed an OR of 3.74 (95\% CI: 2.47-5.66) (Figure 5). The remaining articles were grouped together as other cancer types, showing an OR of 2.92 (95\% CI: 1.16-7.32) (Figure 6).

\section{Discussion}

In the present study, a total of eleven articles were analyzed including a total of 987 histological tissues from patients with different cancer types, showing STn+ expression in

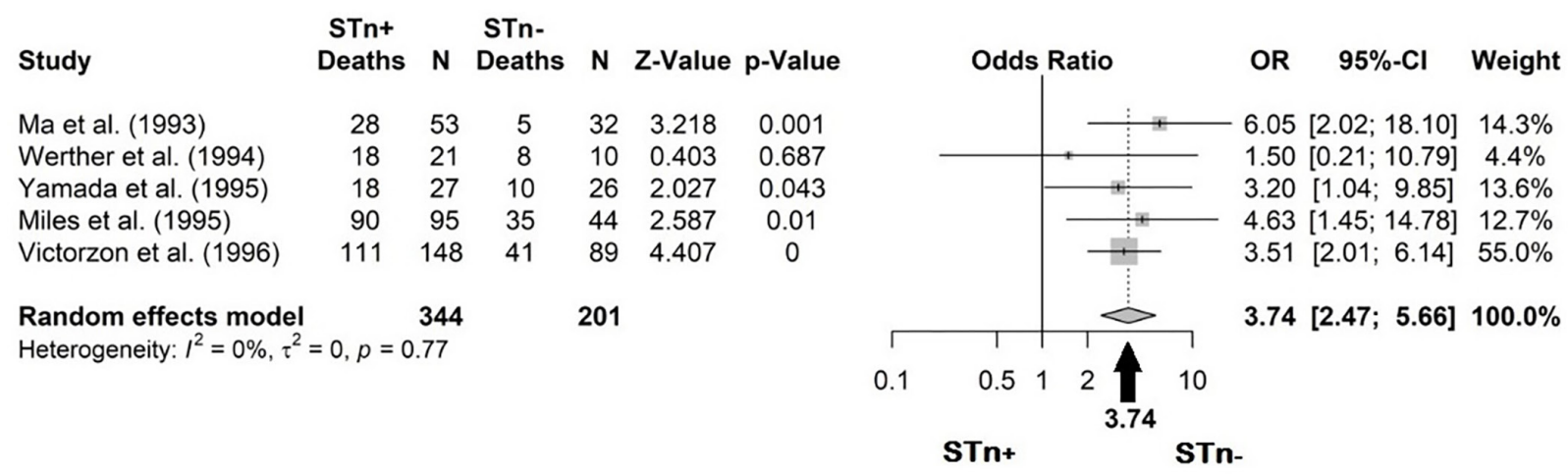

Figure 5 Forest plot of the odds ratio (OR) mortality (STn+vs. STn-) in cancer gastric patients. STn, Sialyl-Tn.

\begin{tabular}{|c|c|c|c|c|c|c|c|c|c|c|c|c|c|}
\hline Study & $\begin{array}{c}\text { STn+ } \\
\text { Deaths }\end{array}$ & $\mathbf{N}$ & $\begin{array}{l}\text { STn- } \\
\text { Deaths }\end{array}$ & $\mathbf{N}$ & Z-Value & p-Value & & Odds & s Ratio & & OR & $95 \%-\mathrm{Cl}$ & Weight \\
\hline Itzkowitz et al. (1990) & 30 & 112 & 0 & 16 & 1.724 & 0.085 & & & & & 12.20 & {$[0.71 ; 209.65]$} & $8.0 \%$ \\
\hline Franchi et al. (1996) & 16 & 21 & 2 & 9 & 2.539 & 0.011 & & & & & 11.20 & {$[1.73 ; 72.30]$} & $14.1 \%$ \\
\hline Terasawa et al. (1996) & 9 & 42 & 7 & 41 & 0.502 & 0.616 & & 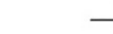 & 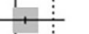 & & 1.32 & {$[0.44 ; 3.97]$} & $22.6 \%$ \\
\hline Ghazizadeh et al. (1997) & 26 & 32 & 2 & 6 & 2.21 & 0.027 & & & 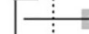 & - & 8.67 & {$[1.28 ; 58.85]$} & $13.6 \%$ \\
\hline Takao et al. (1999) & 39 & 54 & 11 & 13 & -0.906 & 0.365 & & + & - & & 0.47 & {$[0.09 ; 2.39]$} & $16.4 \%$ \\
\hline Costa et al. (2015) & 30 & 57 & 10 & 39 & 2.585 & 0.01 & & & - & & 3.22 & {$[1.33 ; 7.82]$} & $25.4 \%$ \\
\hline \multicolumn{4}{|c|}{$\begin{array}{l}\text { Random effects model } \\
\text { Heterogeneity: } l^{2}=54 \%, \tau^{2}=0.6626, p=0.05\end{array}$} & 124 & & & 1 & 1 & & 1 & 2.92 & {$[1.16 ; 7.32]$} & $100.0 \%$ \\
\hline & & & & & & & 0.01 & 0.1 & 1 & 100 & & & \\
\hline
\end{tabular}

Figure 6 Forest plot of the odds ratio (OR) mortality (STn+ vs. STn-) in other cancer types. STn, Sialyl-Tn. 
662 cases vs. 325 cases of STn- expression. The overall calculation of odds ratio mortality across all the analysed cancer types in this study, suggests that expression of this antigen increases the risk of mortality among several cancer types. This was in accordance with the conclusions of the majority of the individual studies, with the exception of Takao et al. (15) and Terasawa et al. (16) that reported no association between the STn expression and survival, in liver and cervical cancer, respectively. Werther et al. (23), Terasawa et al. (16) and Takao et al. (15) noted that STn reports high sensitivity and recognized it as a one of the best markers of the tumor-associated mucin antigens. Another finding from this systematic review suggests that STn expression could be related to tumor progression in advanced stage of cancers $(5,15,23)$.

Survival analysis in cancer studies is an important tool for assessing the influence of molecular markers on clinical and pathologic features. Similar works have been published assessing the prognostic value of different mucin-associated carbohydrates antigens from serum and/or histological tissues. Liang et al. (26) reported the overexpression of Sialyl LewisX $\left(\mathrm{sLe}^{\mathrm{x}}\right.$ ) on cancer survival in patients reviewing from 8 studies, in this analysis, the statistical heterogeneity obtained was low; however one remarkable limitation was the different sources used to evaluate the expression of $s \mathrm{Le}^{\mathrm{x}}$. Additionally, a non-statistical significance was observed, suggesting that some bias were included during the selection of the articles. Another meta-analysis by Niv (27), exploring the expression of different types of mucins in pancreatic cancer, showed an OR of 10.206, meaning that expression of mucins increases 10 times the risk of mortality in pancreatic cancer, however high heterogeneity was observed in this study. Moreover, Niv did not report on the relationship between mucin types and survival. Although, both studies presented some limitations, still they concluded that the expression of mucins (sLe ${ }^{x}, S T n$ and other) was associated with a poor prognosis and invasion. Furthermore, a literature review by Julien et al. (3) described the expression of STn from different sources as serum samples, cell lines or animal models. However, the vast majority of studies included are only descriptive and present lack of evidence on the prognostic value of STn. In this systematic review, the selection of studies was limited for antibodies TKH2 and B72.3, in order to avoid or reduced differences when comparing the expression of the STn antigen among studies. Both antibodies are the most used and frequent in the scientific literature using histological tissue samples.

A few studies to date have investigated on the association of STn with an increased risk of mortality $(5,8,13,14,21,22)$. Based on our results, positive-STn is related with a strong clinical impact in cancer $(\mathrm{OR}=3.26)$, it shows that survival time was significantly longer in patients with negativeSTn tumors than in those with positive results (Figure 3). The heterogeneity of this study was low $\left(\mathrm{I}^{2}=27 \%\right)$, suggesting a correlation of positive-STn expression with a higher incidence of aggressiveness and decreased survival of patients with different cancer types, across the selected studies.

\section{Conclusions}

To our knowledge this is the first systematic review and meta-analysis that shows evidence of the association between the expression of STn in survival patients from different types of cancers. Results from this study confirm that there is scientific evidence of the positive relation between STn expression and poor prognosis in different types of cancer from histological tissue samples. Currently, research groups are working on undercovering the potential of the STn antigen combined with markers of proliferation (Ki-67) (28), estrogen receptor (ER) (29), and their relationship with circulating tumor cells (CTC) (30) in early cancer diagnosis. In addition, with this new evidence and information, STn has again drawn the attention to be used as a potential candidate for immunotherapy (7), that might lead into the development of novel and effective treatment for cancer patients.

\section{Acknowledgments}

We are very grateful to M.Sc. Julio Vega for helping with the statistical analysis.

Funding: None.

\section{Footnote}

Conflicts of Interest: The authors have completed the ICMJE uniform disclosure form (available at http://dx.doi. org/10.21037/tcr.2019.11.53). The authors have no conflicts of interest to declare.

Ethical Statement: The authors are accountable for all aspects of the work in ensuring that questions related to the accuracy or integrity of any part of the work are appropriately investigated and resolved. The study was approved by Research Committee and the Ethics 
Committee from the Regional High Speciality Hospital of the Yucatan Peninsula (No. CONBIOETICA-31CEI-002-20170731).

Open Access Statement: This is an Open Access article distributed in accordance with the Creative Commons Attribution-NonCommercial-NoDerivs 4.0 International License (CC BY-NC-ND 4.0), which permits the noncommercial replication and distribution of the article with the strict proviso that no changes or edits are made and the original work is properly cited (including links to both the formal publication through the relevant DOI and the license). See: https://creativecommons.org/licenses/by-nc$\mathrm{nd} / 4.0 \%$.

\section{References}

1. Munkley J. The Role of Sialyl-Tn in Cancer. Int J Mol Sci 2016;17:275.

2. Itzkowitz SH, Yuan M, Montgomery CK, et al. Expression of Tn, sialosyl-Tn, and $\mathrm{T}$ antigens in human colon cancer. Cancer Res 1989;49:197-204.

3. Julien S, Videira P, Delannoy P. Sialyl-tn in cancer:(how) did we miss the target? Biomolecules. 2012; 2: 435-66.

4. Pinho S, Marcos NT, Ferreira B, et al. Biological significance of cancer-associated sialyl-Tn antigen: modulation of malignant phenotype in gastric carcinoma cells. Cancer Lett 2007;249:157-70.

5. Victorzon M, Nordling S, Nilsson O, et al. Sialyl Tn antigen is an independent predictor of outcome in patients with gastric cancer. Int J Cancer 1996;65:295-300.

6. MacLean GD, Miles DW, Rubens RD, et al. Enhancing the effect of THERATOPE STn-KLH cancer vaccine in patients with metastatic breast cancer by pretreatment with low-dose intravenous cyclophosphamide. J Immunother Emphasis Tumor Immunol 1996;19:309-16.

7. Bhatia R, Gautam SK, Cannon A, et al. Cancer-associated mucins: role in immune modulation and metastasis. Cancer Metastasis Rev 2019.

8. Itzkowitz SH, Bloom EJ, Kokal WA, et al. SialosylTn. A novel mucin antigen associated with prognosis in colorectal cancer patients. Cancer 1990;66:1960-6.

9. Itzkowitz SH, Marshall A, Kornbluth A, et al. SialosylTn antigen: initial report of a new marker of malignant progression in long-standing ulcerative colitis. Gastroenterology 1995;109:490-7.

10. Kakeji Y, Maehara Y, Morita M, et al. Correlation between sialyl Tn antigen and lymphatic metastasis in patients with Borrmann type IV gastric carcinoma. Br J Cancer 1995;71:191-5.

11. Ikeda Y, Mori M, Kamakura T, et al. Immunohistochemical expression of sialyl Tn and sialyl Lewis(a) antigens in stromal tissue correlates with peritoneal dissemination in stage IV human gastric cancer. Eur J Surg Oncol 1995;21:168-75.

12. Pinto R, Carvalho AS, Conze T, et al. Identification of new cancer biomarkers based on aberrant mucin glycoforms by in situ proximity ligation. J Cell Mol Med 2012;16:1474-84.

13. Ghazizadeh M, Ogawa H, Sasaki Y, et al. Mucin carbohydrate antigens ( $\mathrm{T}, \mathrm{Tn}$, and sialyl-Tn) in human ovarian carcinomas: relationship with histopathology and prognosis. Hum Pathol 1997;28:960-6.

14. Costa C, Pereira S, Lima L, et al. Abnormal Protein Glycosylation and Activated PI3K/Akt/mTOR Pathway: Role in Bladder Cancer Prognosis and Targeted Therapeutics. PLoS One 2015;10:e0141253.

15. Takao S, Uchikura K, Yonezawa S, et al. Mucin core protein expression in extrahepatic bile duct carcinoma is associated with metastases to the liver and poor prognosis. Cancer 1999;86:1966-75.

16. Terasawa K, Furumoto H, Kamada M, et al. Expression of Tn and sialyl-Tn antigens in the neoplastic transformation of uterine cervical epithelial cells. Cancer Res 1996;56:2229-32.

17. Thor A, Ohuchi N, Szpak CA, et al. Distribution of oncofetal antigen tumor-associated glycoprotein-72 defined by monoclonal antibody B72. 3. Cancer Res 1986;46:3118-24.

18. Loureiro LR, Carrascal MA, Barbas A, et al. Challenges in antibody development against $\mathrm{Tn}$ and sialyl-Tn antigens. Biomolecules 2015;5:1783-809.

19. Wells GA SB, O'Connell D, Peterson J, Welch V, Losos M. . The Newcastle-Ottawa Scale (NOS) for assessing the quality of nonrandomised studies in meta-analyses. 2018. Available online: http://www.ohri.ca/programs/clinical_ epidemiology/oxford.asp.

20. Sterne JA, Egger M. Funnel plots for detecting bias in meta-analysis: guidelines on choice of axis. J Clin Epidemiol 2001;54:1046-55.

21. Ma XC, Terata N, Kodama M, et al. Expression of sialylTn antigen is correlated with survival time of patients with gastric carcinomas. Eur J Cancer 1993;29a:1820-3.

22. Yamada T, Watanabe A, Yamada Y, et al. Sialosyl Tn antigen expression is associated with the prognosis of patients with advanced gastric cancer. Cancer 
1995;76:1529-36.

23. Werther JL, Rivera-MacMurray S, Bruckner H, et al. Mucin-associated sialosyl-Tn antigen expression in gastric cancer correlates with an adverse outcome. Br J Cancer 1994;69:613-6.

24. Miles D, Linehan J, Smith P, et al. Expression of sialylTn in gastric cancer: correlation with known prognostic factors. Br J Cancer 1995;71:1074.

25. Franchi A, Gallo O. Prognostic implications of Sialosyl-Tn antigen expression in sinonasal intestinaltype adenocarcinoma. Eur J Cancer B Oral Oncol 1996;32b:123-7.

26. Liang JX, Liang Y, Gao W. Clinicopathological and prognostic significance of sialyl Lewis $\mathrm{X}$ overexpression in

Cite this article as: Lugo R, Ávila-Nava A, García-Pérez R, Herrera-Escalante S, De la Cruz-Acosta J, Gutiérrez-Solis AL. Systematic review and meta-analysis of the clinical survival significance of Sialyl-Tn expression in histological tissues from cancer patients. Transl Cancer Res 2020;9(2):547-555. doi: $10.21037 /$ tcr.2019.11.53 patients with cancer: a meta-analysis. Onco Targets Ther 2016;9:3113-25.

27. Niv Y. Mucin expression and the pancreas: A systematic review and meta-analysis. World J Meta-Anal 2017;5:63-70.

28. Ferreira JA, Videira PA, Lima L, et al. Overexpression of tumour-associated carbohydrate antigen sialyl-Tn in advanced bladder tumours. Mol Oncol 2013;7:719-31.

29. Leivonen M, Nordling S, Lundin J, et al. STn and prognosis in breast cancer. Oncology 2001;61:299-305.

30. Lima L, Severino P, Silva M, et al. Response of high-risk of recurrence/progression bladder tumours expressing sialyl-Tn and sialyl-6-T to BCG immunotherapy. Br J Cancer 2013;109:2106. 
Supplementary

Table S1 Newcastle Ottawa scale for assessment of quality of included studies

\begin{tabular}{|c|c|c|c|c|}
\hline Studies & Selection & Comparability & Outcome & Conclusion \\
\hline Ma et al. (1993) & 论标 & 拧 & 论论坛 & Fair \\
\hline Werther et al. (1994) & 论标 & 论标 & 论柁 & Fair \\
\hline Yamada et al. (1995) & 论许访 & 论弥 & 论动 & Good \\
\hline Yamada et al. (1995) & 论败访 & 论弥 & 论弥 & Good \\
\hline Victorzon et al. (1996) & 论动 & 访 & 论论该 & Fair \\
\hline Itzkowitz et al. (1990) & 论论访 & 论弥 & 论拧 & Good \\
\hline Takao et al. (1999) & 论论坛 & 论放 & 论弥 & Good \\
\hline Costa et al. (2015) & 论论坛 & 访 & 论动 & Fair \\
\hline Ghazizadeh et al. (1997) & 论败许 & 论 & 论弥 & Fair \\
\hline Terasawa et al. (1996) & 论败访 & 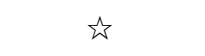 & 论弥 & Fair \\
\hline Franchi et al. (1996) & 访 & 论标 & 论弥访 & Good \\
\hline
\end{tabular}

is represents if individual criterion within the subsection was fulfilled. A study can be awarded a maximum of four stars for selection; two stars for comparability and three stars for outcome. The total score of the studies were rated as good, fair, or poor quality. Thresholds for converting the Newcastle-Ottawa scales to AHRQ standards (good, fair, and poor) (19): Good quality: 3 or 4 stars in selection domain AND 1 or 2 stars in comparability domain AND 2 or 3 stars in outcome/exposure domain. Fair quality: 2 stars in selection domain AND 1 or 2 stars in comparability domain AND 2 or 3 stars in outcome/exposure domain. Poor quality: 0 or 1 star in selection domain OR 0 stars in comparability domain OR 0 or 1 stars in outcome/exposure domain. 